\title{
Autoarchivo de artículos biomédicos en repositorios de acceso abierto
}

\author{
M. Francisca Abad-García, Remedios Melero, Ernest Abadal, Aurora González-Teruel
}

Introducción y desarrollo. El acceso abierto es el acceso digital, en línea y libre de barreras económicas, o al menos de algunos derechos de explotación (copyright), a la producción científica o académica de nuestros profesores e investigadores. El autoarchivo o depósito de estos trabajos en repositorios institucionales (vía verde) está cada vez más presente entre las actividades de la comunidad científica. Además de beneficiarse de sus ventajas, los autores son, cada vez con más frecuencia, requeridos por las instituciones financiadoras de la investigación y por las suyas propias para que depositen sus trabajos en repositorios. En el entorno biomédico esto es más necesario, por la repercusión que puede tener en la salud de las personas que los profesionales dispongan o no de la mejor evidencia científica. Para que esto sea posible, los autores deben conocer qué es y cómo funciona un repositorio y deben saber manejar herramientas fáciles como Sherpa/ RoMEO o DULCINEA para averiguar qué derechos retienen como autores y si éstos les permiten llevar a la práctica el autoarchivo. PubMed Central y sus homólogos británico y canadiense son los principales repositorios temáticos del ámbito biomédico. En nuestro país no existe ninguno de naturaleza similar; sin embargo, la mayoría de las universidades y el Consejo Superior de Investigaciones Científicas disponen de un repositorio institucional en funcionamiento.

Conclusión. La mayor visibilidad de los resultados de investigación y su repercusión en una mayor y más temprana citación es uno de los beneficios más esgrimidos del acceso abierto, así como la eliminación de las barreras económicas en el acceso a la información contrastada para colectivos habitualmente excluidos.

Palabras clave. Acceso abierto. Autoarchivo. Open access. Publicaciones biomédicas. Repositorios.

\section{Introducción}

Es cada vez más frecuente que en los requisitos de las convocatorias de ayudas para la investigación [14] se mencione la recomendación o el requisito del autoarchivo o del depósito de los trabajos científicos en repositorios institucionales o temáticos. También comienza a ser habitual que las editoriales asuman políticas que permitan la reutilización posterior de los artículos publicados en sus revistas. Estas situaciones, entre otras, son consecuencia de lo que se conoce como acceso abierto a la ciencia, que es la traducción a nuestro idioma de la expresión anglosajona open access. La repercusión del open access en las publicaciones científicas y en el modo en el que se difunde actualmente la ciencia es enorme, lo que ha provocado que sus características se vean reflejadas no sólo en la bibliografía profesional [5], sino también en la biomédica, tanto de ámbito nacional [6-12] como, sobre todo, internacional, como ponen de manifiesto las cerca de 300 publicaciones sobre el tema que recoge la base de datos PubMed.

El open access es un movimiento de aparición reciente sobre el que muchos investigadores aún no han oído hablar o del que no conocen su significa- do, pero del que disfrutan de alguna de sus ventajas. Así ocurre cuando al hacer una búsqueda en Google académico recuperan documentos a texto completo que han sido depositados por un científico en su web personal o en un repositorio o que han sido publicados en una revista de acceso abierto. Lo mismo sucede cuando al utilizar la base de datos PubMed recuperan los textos completos de artículos que están depositados en PubMed Central, el mayor repositorio de acceso abierto del ámbito biomédico (Fig. 1).

Pero, ¿qué es el acceso abierto? Para definirlo es habitual tomar como referencia las declaraciones de las tres reuniones que tuvieron lugar entre los años 2002 y 2003 en Budapest, Bethesda y Berlín [13-15], y que pueden considerarse como 'fundacionales' de este movimiento. Éstas establecen este movimiento en torno a dos principios: gratuidad en el acceso a la información y retención al menos de algunos derechos de autor que permitan la reutilización de sus obras y la cesión de su uso a terceros. De acuerdo con esto, se entiende por open access: 'El acceso digital, en línea y libre de barreras económicas o de copyright a los trabajos publicados con revisión por pares por la comunidad científica. Esto
Departamento de Historia de la Ciencia y Documentación; Facultad de Medicina; Universitat de València (M.F. Abad-García, A. González-Teruel). Instituto de Agroquímica y Tecnología de Alimentos; CSIC; Burjasot, Valencia (R. Melero). Departamento de Biblioteconomía y Documentación; Universitat de Barcelona; Barcelona, España (E. Abadal).

Correspondencia:

Dra. M. Francisca Abad García. Departamento de Historia de

la Ciencia y Documentación.

Facultad de Medicina. Universitat de València. Blasco Ibáñez, 15.

E-46010 Valencia.

Fax:

+34963864091.

E-mail:

abad@uv.es

Aceptado tras revisión externa: 11.11.09.

Cómo citar este artículo: Abad-Carcía MF, Melero R, Abada E, González-Teruel A. Autoarchivo de artículos biomédicos en repositorios de acceso abierto. Rev Neurol 2010; 50: 431-40.

(c) 2010 Revista de Neurología 
Figura 1. Acceso a artículos depositados en el repositorio de acceso PubMed Central a través de la búsqueda en la base de datos PubMed.

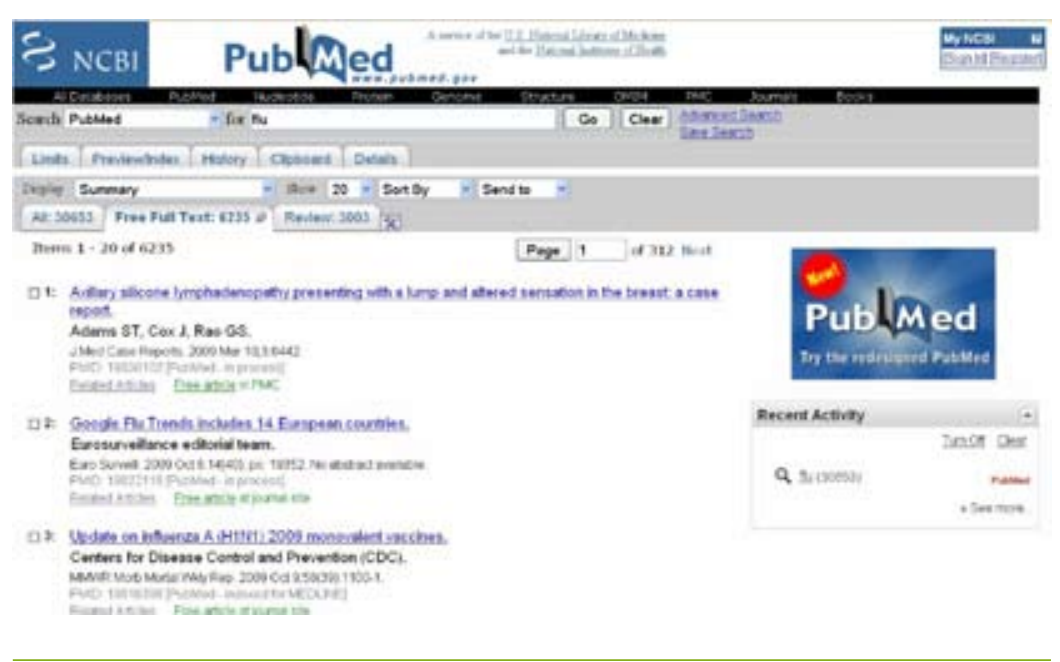

es, gratuidad y posibilidad de leer, descargar, copiar, distribuir, imprimir los textos científicos y utilizarlos para cualquier otro propósito siempre que sea dentro de la ley'

'Para que esto sea posible es condición que el autor(es) como titular(es) de los derechos de autor garantice(n) a los usuarios el derecho perpetuo, irrevocable, mundial, de acceder, copiar, usar, distribuir, transmitir y mostrar la obra públicamente y hacer y distribuir obras derivadas, en cualquier medio digital para cualquier propósito dentro de la ley, siempre sometido a la adecuada atribución de la autoría, así como el derecho a hacer un pequeño número de copias impresas para su uso personal.'

Adicionalmente, en las reuniones anteriormente mencionadas también se establecieron cuáles eran las vías para la materialización del acceso abierto. Una, a la que se denominó vía dorada por ser la más directa e inmediata, sería la publicación de artículos en revistas de acceso abierto; y la otra, denominada verde, mucho más posibilista, estaría constituida por el depósito o archivo de copias de los artículos en bases de datos específicas, denominadas repositorios.

La reivindicación de acceso abierto a la información que se traduce de estas declaraciones es ante todo un ejercicio de coherencia entre la forma actual de trabajo de los científicos, basada en las redes de colaboración y comunicación, y el modo en el que se quiere que fluya la información científica. Es una reivindicación que colisiona frontalmente con el mecanismo utilizado hasta el momento para el acceso a la información, sustentado en el acceso restringido a los contenidos de las revistas científicas mediante el pago de suscripciones personales o institucionales a las editoriales.

Aunque ha sido en el siglo XXI cuando nos hemos familiarizado con este movimiento, su origen se remonta a principios de los años noventa, y en él convergen varias circunstancias. Sin duda, la más mencionada ha sido el encarecimiento de las suscripciones de las revistas científicas, cuyos precios han superado en los últimos 15 años en más de un $150 \%$ a la tasa de inflación, mientras que los presupuestos de las bibliotecas permanecían estables o a la baja [16]. Esto ha supuesto, entre otras cosas, que muchas instituciones que generan gran parte de los contenidos de las revistas no las puedan suscribir, y también que se hayan roto las expectativas de que la introducción de las tecnologías de información (que pueden simplificar y abaratar el trabajo editorial) repercutan en un aumento de disponibilidad de información.

Esta situación, que en otro momento hubiera resultado sólo en una restricción de fondos para las bibliotecas, se ha producido en un período en el que Internet ha creado una cultura de acceso y uso de la información que hace difícil imaginar a la comunidad científica resignada a aceptar las barreras económicas que impone el mercado editorial para el acceso a la información generada por sus pares, barreras cuyo origen reside en la cesión de los derechos de explotación comercial de los trabajos ( $\mathrm{co}$ pyright) que las editoriales imponen a los autores como requisito para la publicación de sus trabajos.

Un aspecto clave a este respecto ha sido la consideración de la financiación y, por ende, de la propiedad de aquello que el autor está cediendo a las editoriales. Así, si se tiene en cuenta que, al menos en el sector biomédico, la mayoría de los investigadores desarrolla su labor en centros públicos (hospitales, universidades, centros de investigación) y que buena parte de la investigación recibe financiación adicional de fondos públicos mediante becas, ayudas y subvenciones de diversa naturaleza, resulta que, por una parte, el autor está cediendo los derechos de comercialización de algo que no es enteramente suyo y que, además, se está pagando con dinero público dos veces por el mismo producto: una vez para realizar la investigación y otra para poder acceder a sus resultados.

Este argumento está siendo esgrimido cada vez con más frecuencia por las instituciones que apoyan el movimiento de acceso abierto. Así, en su papel de productoras de investigación y, en ocasio- 
nes, financiadoras de ésta, cuestionan la cesión exclusiva de los derechos que hasta el momento han hecho los autores de forma unilateral, situándose en una posición de copartícipes de los resultados, lo que les permite exigir determinadas condiciones mediante la adopción de políticas institucionales.

En el ámbito biomédico, la repercusión que puede tener para la salud de las personas el que los profesionales dispongan o no de la mejor evidencia científica disponible, la magnitud que supone la inversión en investigación en materia sanitaria, junto con la repercusión que en términos de mejora sanitaria puede suponer para los países pobres el acceso a la ciencia sin barreras económicas han sido, entre otros, los motivos que sin duda han impulsado a las instituciones al desarrollo temprano, y contundente en muchas ocasiones, de políticas a favor del acceso abierto, unas de recomendación y otras de requisito, que establecen que las publicaciones resultantes de la investigación que financian deben estar disponibles en acceso abierto en un plazo de tiempo determinado.

Uno de los países que más se ha implicado en esas políticas ha sido el Reino Unido. De hecho, durante el período 2007-2008, de los 1,2 millardos de libras que dedicaron a la financiación de la investigación los UK Research Councils, 0,76 millardos $(61,3 \%)$ fueron fondos de ayuda a la investigación cuya convocatoria estaba realizada con una política de acceso abierto. De éstos, 0,4 millardos (55,5\%) correspondían a los fondos de financiación de los Medical Research Councils y del Biotechnology and Biological Research Council [17]. En estos momentos, nueve instituciones financiadoras de investigación en materia biosanitaria del Reino Unido poseen una política de acceso abierto. Canadá es otro de los países que más han desarrollado políticas en este sentido, y cuenta en materia específicamente sanitaria con 12 [18]. Por su parte, Estados Unidos también ha sido un país que ha desarrollado políticas de acceso abierto, como, por ejemplo, la del Howard Hughes Medical Institute o la contundente de los National Institutes of Health (NIH). Así, en el año 2005, los NIH instaban a sus investigadores al depósito de sus trabajos en un plazo no superior a 12 meses desde su publicación En abril de 2008, esta solicitud se transformó de recomendación en requisito. Esta política es, sin duda, una de las ejemplares, no sólo por su contundencia, sino por la cantidad de dinero que implica y la cantidad de publicaciones resultantes de la investigación financiada que estarán disponibles en acceso abierto. Esta política no ha sido del total agrado del sector editorial, que ve en estas medidas una amenaza para su mercado, algo que ha generado la creación de lobbies que en su momento encontraron apoyo entre miembros del Congreso para lanzar una proyecto de ley para derogar la política de los NIH, si bien, por ahora, no han contado con el apoyo suficiente para continuar su curso. Actualmente, el Senado de Estados Unidos todavía está por resolver el proyecto de ley Federal Research Public Access Act of 2009, en el cual se solicita que todos los proyectos financiados por agencias federales, entre las cuales esta el $\mathrm{NIH}$, establezcan políticas de acceso abierto a las publicaciones que se deriven de ellos $[19,20]$.

A nivel más general, en 2005, la Organización para la Cooperación y el Desarrollo Económico [21], la International Federation of Library Association y la UNESCO [22] realizaron declaraciones que instaban a la comunidad científica y a los estados miembros a avalar el paradigma del acceso abierto como vía de difusión del conocimiento y generación de desarrollo. En esta línea, las principales agencias de investigación internacionales se están sumando a la adopción de políticas de acceso abierto vinculadas a la concesión proyectos. Es el caso de Alemania, Australia, Austria, Bélgica, Canadá, Escocia, Francia, Hungría, Irlanda, Noruega, Suecia y Suiza, entre otros.

En un ámbito supranacional, en enero de 2008, la Comisión Europea [23] anunció un ensayo piloto por el cual el $20 \%$ del total de los fondos dedicados a financiar proyectos en investigación del séptimo programa marco (FP7) deberían cumplir el requisito de hacer que las publicaciones derivadas de ellos fueran de acceso abierto en un plazo no superior a seis meses desde su publicación en el caso de las áreas relacionadas con la energía, el medio ambiente, la salud, las tecnologías de la información y la comunicación, y las infraestructuras de la investigación, o de 12 meses para ciencia y sociedad, ciencias sociales y humanidades. Esta política es una política ejemplar, ya que es un mandato, no una recomendación, de alcance europeo. La importancia de esta política también tiene cifras: el European Research Council desembolsará 7,5 billones de euros, que son aproximadamente el 15\% del presupuesto para la investigación de la Unión Europea en el FP7.

En España, las únicas políticas existentes son las de las recientes convocatorias de proyectos de la Comunidad de Madrid [1], las del Consejo Superior de Investigaciones Científicas (CSIC) [2] y las de las universidades madrileñas Rey Juan Carlos I y Carlos III [3-4]. Asimismo, cabe mencionar también la política de acceso abierto del Principado de Asturias, por la 
que se requiere el depósito de las publicaciones que recojan los resultados de cualquier investigación que haya recibido ayudas o subvenciones procedentes de fondos públicos gestionados u otorgados por la Administración del Principado de Asturias.

\section{¿Qué es un repositorio?}

Un repositorio es una base de datos que posee unas características especiales. La primera es que no aporta sólo información referencial, sino que almacena objetos digitales que pueden ser de diversa naturaleza (textuales, de imagen o sonido). La segunda es que en la base de datos cada recurso $u$ objeto digital está identificado y caracterizado por un conjunto de metadatos estandarizados. Estos metadatos proporcionan información descriptiva (autor, título, materias, etc.), de administración del recurso (creación del recurso, derechos, control de acceso...) y de preservación (tipo de formato, etc.), y son los que facilitan su recuperación selectiva en Internet. Para permitir la interoperabilidad del repositorio y la posibilidad de acceder libremente a sus contenidos [24], la base de datos está diseñada con software libre que utiliza un determinado protocolo denominado OAI-PMH (Open Access Initiative-Protocol Metadata Harverster). Además, los recursos incluidos en el repositorio están asociados a una URL que es única, lo que permite la localización permanente del recurso.

Habitualmente, los repositorios se clasifican según la procedencia de los documentos que contienen. De este modo, se habla de repositorios institucionales cuando los objetos digitales que contienen reflejan la producción científica de una institución determinada; cooperativos o centrales, cuando corresponden a varias instituciones; o temáticos, cuando el depósito de objetos digitales está vinculado no a la procedencia institucional de aquél que deposita, sino a la temática de su contenido.

Pese a que los objetivos específicos de uno u otro tipo de repositorio pueden variar, en líneas generales podrían resumirse en los tres siguientes:

- Recoger y preservar los resultados de la investigación en formato digital, garantizando su permanencia en el tiempo.

- Abrir y ofrecer a la comunidad científica y a la sociedad en general los resultados de las investigaciones realizadas por miembros de la institución o de aquéllos que trabajan en un determinado ámbito temático.

- Aumentar el impacto y la influencia de los resultados, maximizando la visibilidad de los resultados.

\section{¿Qué tipo de documentos pueden depositarse en un repositorio?}

Pese a que el movimiento de acceso abierto en un primer momento centra su atención sobre todo en la libre disponibilidad de artículos científicos publicados en revistas con revisión por pares, cada vez es más frecuente que se depositen en los repositorios objetos digitales de distinta naturaleza y finalidad. Así, no es extraño que en repositorios universitarios se depositen todo tipo de documentos generados en el ámbito de la institución en el cumplimiento de sus funciones docente, investigadora y de difusión de la cultura. Encontramos, pues, artículos científicos junto con tesis doctorales o, por ejemplo, junto con objetos de aprendizaje orientados a la actividad docente. Según la institución, también encontramos repositorios con un contenido puramente cultural [25-27], e incluso administrativo [28]. Así, es posible que los repositorios, además de por su ámbito, puedan clasificarse por el tipo de contenido, e incluso por su versión. No obstante, la distinción que prevalece es entre repositorios institucionales y temáticos.

\section{¿Quién incorpora los documentos al repositorio?}

El mecanismo de obtención de los documentos que deben estar incluidos en un repositorio es distinto al de las bases de datos bibliográficas a las que estamos acostumbrados, en las que la institución o el organismo que las confecciona obtiene las referencias de los trabajos que aparecen publicados en un conjunto de revistas que conforman su cobertura. Así, el funcionamiento de un repositorio se plantea de un modo distinto.

En términos ideales, deberían ser los propios autores los que tendrían que introducir los documentos y los metadatos que los describen, motivo por el que en la bibliografía sobre acceso abierto se denomina a este proceso autoarchivo. Éste es el modo en el que funcionan algunos repositorios, sobre todo de tipo temático, que están impulsados por colectivos de científicos que son conocedores de las ventajas y ganancias que implica que su producción científica sea de acceso abierto. Es el caso, por ejemplo, de ArXiv [29], repositorio de física, creado en 1991 y que constituye en esta rama del conocimiento un referente para la comunicación y el intercambio de ideas y resultados, o E-Lis, el repositorio de biblioteconomía y documentación [30]. Cuando un repositorio funciona mediante el 
autoarchivo, los autores deben estar dados de alta en el sistema para poder introducir documentos, y suele existir el papel de un administrador del sistema o de un comité que ejerce esas funciones, con la finalidad de asegurar la no incorporación de contenidos inapropiados.

Sin embargo, en estos momentos en los que la mayoría de repositorios comienzan su andadura y en los que aún son muchos los investigadores que desconocen su propósito, es frecuente que se realice un depósito delegado o que se utilice un procedimiento mixto (autoarchivo + depósito delegado). El depósito delegado suelen llevarlo a cabo las unidades y servicios a cargo de la gestión y mantenimiento del repositorio, que en la mayoría de las instituciones son las bibliotecas. Estos depósitos delegados se realizan poniendo en práctica procedimientos que facilitan la localización y captura de documentos que deben incluirse en el repositorio. Así, por ejemplo, estas unidades localizan la producción científica de los investigadores y les solicitan copias en formato digital de sus trabajos para introducirlos en el repositorio, o bien los autores voluntariamente hacen llegar a las bibliotecas sus trabajos para que éstos sean depositados.

También se da la circunstancia de que el depósito sea mediado por la propia revista en la que publica en al autor, como en el caso de las revistas que depositan total o parcialmente sus artículos en PubMed Central.

\section{Querer no es poder. ¿Qué ha de \\ tenerse en cuenta antes de incorporar un documento a un repositorio?}

Para depositar un trabajo en un repositorio, no sólo hay que querer hacerlo, sino que poder hacerlo, de tal modo que para poder hacerlo deben cumplirse una de las dos condiciones siguientes: el autor es el poseedor de los derechos de explotación comercial (copyright) del documento, o bien el autor no posee esos derechos, pero su propietario lo permite. En el primer supuesto estarían, por ejemplo, las tesis doctorales, algunos informes de investigación o los artículos publicados en revistas de acceso abierto. En el segundo supuesto, deberá averiguarse si el autoarchivo está o no permitido antes de llevarlo a cabo. Además, en el caso de los repositorios institucionales para poder depositar, el autor debe pertenecer a la institución en cuestión.

En el caso de los artículos científicos, que los autores puedan depositar sus trabajos depende de las condiciones de copyright que establezcan las editoriales (véanse, por ejemplo, las condiciones de Elsevier [31]). La mayoría de revistas que no son de acceso abierto requieren como condición para la publicación de un artículo que los autores renuncien por escrito a sus derechos de propiedad (se renuncia a todos los derechos menos al derecho moral de reconocimiento de autoría) de forma permanente a favor de la editorial, permitiendo que ésta realice una explotación comercial de la obra. Así, los derechos de reproducción, copia y difusión quedan en poder de la editorial de la revista, y el autor podrá disponer de su trabajo sólo en los términos que la editorial establezca, lo que también afecta al autoarchivo. Estas condiciones afectan también a las revistas que, sin ser de acceso abierto, han decidido que sus artículos sean consultables libremente en Internet (conocidas como free access), ya que son revistas en las que el autor ha transferido los derechos de explotación a la editorial. Sería el caso de revistas médicas muy conocidas en el ámbito biomédico español como es el caso de Atención Primaria, Gaceta Sanitaria o Neurología.

Para averiguar si podemos o no autoarchivar un artículo, qué versión podemos autoarchivar, cuándo y dónde podríamos consultar la información que proporcionan las revistas, las editoriales, ante la evolución del movimiento de acceso abierto, poco a poco van incorporando estos datos en el apartado de copyright, o a veces en las normas para los autores. Sin embargo, aún no es muy frecuente que esta información esté disponible y su localización no es siempre sencilla.

Por ello, para facilitar esta tarea puede consultarse la web de Sherpa/RoMEO (Fig. 2) [32], que es un servicio gestionado por la Universidad de Nottingham, con financiación del JISC y de la Wellcome Trust, que proporciona información sobre las políticas de copyright y de autoarchivo de las editoriales. En estos momentos, Sherpa/RoMEO proporciona información sobre más de 600 editoriales, que agrupan más de 9.000 revistas científicas de ámbito internacional. Consultando esta web, bien por nombre la revista o de su editorial, podremos averiguar qué versión del documento puede depositarse (pre-print o post-print), el momento del autoarchivo (tras la aceptación del trabajo, tras la publicación o pasado un período de embargo), el tipo de repositorio (institucional y/o temático) y si la versión del documento debe ser la versión manuscrita del autor o puede utilizarse el pdf de la editorial. De acuerdo con estos datos, la web Sherpa/RoMEO clasifica las editoriales y las revistas en cuatro colores: blanco si no permite el autoarchivo de ninguna versión; amarillo en el caso sólo del pre-print, que 
Figura 2. Descripción de las condiciones de autoarchivo de los artículos de la revista Neurobiology of disease en la base de datos Sherpa/RoMEO.

\section{SHERPA ROMEO}



la salud. En este ámbito, la política de la editorial Elsevier para las revistas sanitarias españolas (antes editadas por Doyma) es predominante, y es remarcable que permite el autoarchivo de la versión postprint del artículo (son revistas azules) en el momento de aceptación del trabajo, por lo que si esto se lleva a cabo, se produce un adelanto, muchas veces considerable, en la fecha en la que los resultados de la investigaciones se hacen visibles.

\section{Poder no es querer. Los autores y el depósito de trabajos en los repositorios}

Llenar los repositorios de contenidos es uno de los retos a los que se enfrentan estas nuevas estructuras de comunicación. Que esto suceda supone un compromiso activo de la comunidad científica, tanto de las instituciones como de los investigadores a título individual. Así, además de poder archivar, los autores deben querer hacerlo. El porqué de este aserto lo encontramos en la reticencia de los autores a la hora de depositar, pues, aunque parezca paradójico, al ser sin duda los mayores beneficiarios de que sus trabajos estén accesibles, constituyen la pieza más difícil de integrar en el sistema.

Conseguir que los autores depositen sus trabajos no es tarea fácil, sobre todo porque el desconocimiento que los científicos tienen de los objetivos que se persiguen con el acceso abierto y de sus procedimientos aún es grande. Además de esto, diversos estudios [36-38] han puesto de manifiesto que el autoarchivo puede verse coartado como consecuencia de la falta de tiempo y de recursos, y también es muy frecuente que éste no se produzca como resultado de la resistencia a nuevas formas de trabajo, actitud que parece ser inherente a la introducción de cualquier cambio. Otros autores no son proclives al depósito por temor a la pérdida de control de su obra y de su trabajo, ya que piensan que, al estar éste disponible en Internet, cualquiera puede apropiarse de su trabajo y piratear sus contenidos. No son así conscientes de que la visibilidad es también una protección contra estos actos, pues cuanto más visible es una obra, más fácilmente puede detectarse el plagio. El temor a entrar en conflicto con el editor y que éste vete la publicación de sus trabajos es también un temor extendido, si bien el uso de instrumentos como RoMEO y DULCINEA puede ayudar a minimizarlo. Por último, otro motivo para no depositar es el desacuerdo con el movimiento de acceso abierto y, en definitiva, el desacuerdo con la idea de compartir el trabajo, algo difícil de combatir con argumentos racionales. 
Como ya se ha comentado, esta situación de falta de disposición al autoarchivo mejora drásticamente con la adopción de mandatos de las agencias financiadoras de la investigación, si bien, en nuestro país, el desarrollo de políticas es aún un tema pendiente que avanza muy despacio. De hecho, el que los NIH pasaran de solicitar el depósito de trabajos a requerirlo supuso en su día elevar drásticamente la tasa de depósito lograda en el repositorio PubMed Central. En ausencia de políticas nacionales de las agencias financiadoras, las instituciones deben elaborar las suyas propias, instando a sus miembros a depositar, adoptando para ello acciones que abarquen desde reglamentaciones que obliguen al depósito de toda la información generada por miembros de la institución, hasta discriminar positivamente el hecho de que los trabajos estén depositados en el caso de promociones o valoración de la investigación en el contexto de la carrera profesional, o incentivar positivamente el depósito, por ejemplo, mediante una pequeña compensación económica asociada a la memoria anual de investigación. Lo que queda claro es que todo ello cobra más sentido si cualquiera de las acciones anteriormente mencionadas se acompaña de adecuadas campañas de información, difusión, promoción y formación, iniciativas que suelen acogerse de mejor grado que las imposiciones.

\section{Dónde depositar. Los repositorios en el área de ciencias de la salud}

Para conocer cuál es el desarrollo actual de los repositorios en el área de biomedicina y de ciencias de la salud, la única fuente consultable es el directorio OpenDoar [39] de la Universidad de Nottingham, al ser el único que permite filtrar los repositorios según el área temática de los contenidos. Según este directorio, hay más de 1.612 repositorios censados, que son, sobre todo, institucionales, pertenecientes a universidades y centros de investigación, siendo los temáticos mucho menos numerosos.

Cuando se considera la categoría health and medicine, el número de repositorios se reduce a 114 . Sin embargo, cuando se repasa uno a uno estos repositorios, se aprecia que en esta categoría se encuentran tanto aquéllos que son multitemáticos, pero que incluyen la medicina y la salud entre los temas, como agrupaciones de revistas que no son repositorios, o repositorios específicamente de contenido biomédico. Si nos centramos en estos últimos, su número asciende únicamente a 34 , de los que 20 serían institucionales y 14 temáticos.
Los pertenecientes a instituciones corresponden, sobre todo, a instituciones académicas, facultades o escuelas en las que se imparten titulaciones relacionadas con las ciencias de la salud (11 repositorios), a centros de investigación sanitaria y biomédica (tres repositorios) o a la administración sanitaria (dos repositorios). Sólo cuatro repositorios corresponden a un centro asistencial.

En cuanto a los repositorios temáticos, llama la atención dos cosas, que a excepción de los dos grandes repositorios temáticos PubMed Central y UK PubMed Central, que incorporan artículos de todos los ámbitos de biomedicina y que contienen actualmente cerca de 1.500.000 documentos, el resto de repositorios suele abarcar temáticas muy concretas (es el caso de Aphasiology Archive [40]; del Archivo Aperto di Documenti per la Medicina Sociale [41]; o del Canadian Breast Cancer Researh Alliance Open Access Archive [42]), y el número de documentos depositados no excede de un millar, o a veces menos.

Si se observan estos datos a la luz de las posibilidades del depósito de la literatura biomédica en el ámbito internacional, puede deducirse que ésta habitualmente se está depositando en los repositorios pertenecientes a universidades, facultades o escuelas en las que se imparten titulaciones relacionadas con las ciencias de la salud, en PubMed Central [43] o UK PubMed Central [44], o en el recién estrenado PubMed Central para Canadá [45]. Esto último está en total sintonía con que la mayoría de las políticas establecidas por las agencias financiadoras de la investigación sanitaria de países como Estados Unidos, Reino Unido o Canadá especifican concretamente que PubMed Central será el repositorio preferente para realizar el depósito.

En nuestro país, de acuerdo con el directorio Busca Repositorios [46], en el momento actual existen 60 repositorios en funcionamiento, la mayoría de ellos vinculados a universidades e instituciones de investigación. El único repositorio con un contenido exclusivamente biomédico es el repositorio de la Sociedad Española de Neuropsiquiatría, creado en 2009 [47]. Por el momento, no existen repositorios en funcionamiento vinculados a ningún centro hospitalario y, por lo que parece, la tendencia en nuestro país va en la línea de la implantación de repositorios centrales vinculados a las bibliotecas virtuales de salud creadas en las comunidades autónomas. Dada la ausencia de repositorios específicos en el momento actual, el depósito de trabajos pueden hacerlo efectivo aquellos profesionales que están vinculados a instituciones universitarias o de investigación que ya tengan repositorios. De 
hecho, son ya muchas universidades las que disponen de un repositorio o están en fase de implantación. También el CSIC tiene un repositorio [48] que agrupa la investigación de todos sus centros.

\section{Beneficios de depositar los trabajos en un repositorio de acceso abierto}

Tal vez el aumento de la visibilidad de los resultados de la investigación sea el efecto beneficioso más esgrimido en defensa del movimiento de acceso abierto y del depósito de los artículos en un repositorio. La publicación de trabajos en una revista que sea sólo accesible mediante suscripción encierra los resultados de la investigación al ámbito de aquéllos que poseen las claves para su consulta. De forma opuesta, un artículo depositado en un repositorio o publicado en una revista de acceso abierto será accesible y visible para todo el mundo, y sitúa los resultados de la investigación en lo que se empieza a conocer como la 'nube' de Internet, o a sólo un clic de Google académico, el buscador más utilizado para la localización de documentación científica. Paralelamente, la mayor visibilidad tiene efectos positivos en la citación de los trabajos [49-51], tanto en el incremento del número de citas recibidas [5256] como en la disminución del tiempo trascurrido en la aparición de las citas [57].

En un terreno más cualitativo, el acceso abierto a los resultados de investigación supone una armonización con las formas de trabajo actual en la red y una eliminación de las barreras económicas que supone el mercado editorial para el acceso a la ciencia en los países pobres. Esto, que es de gran trascendencia en todas las áreas de la ciencia, cobra especial relevancia en el contexto de la medicina, en tanto que disponer de la información adecuada en el momento en la que se requiere puede suponer la diferencia entre la vida y la muerte de pacientes concretos y reducir la morbilidad o sus complicaciones. Es, además, una vía indiscutible para el impulso de la medicina basada en la evidencia y una fuente de información de gran valor para los estudiantes de las ciencias biomédicas, a los que se les permite el uso de la información para el aprendizaje.

El acceso abierto supone, además, una vía de que la información de calidad contrastada (artículos con revisión por pares) esté disponible para otros colectivos de usuarios habitualmente excluidos de ella, como los pacientes y los ciudadanos en general.

Las oportunidades que brinda el acceso abierto están esbozadas en los párrafos anteriores, si bien, dada la juventud de esta iniciativa, muchas de sus vertientes permanecen sin explorar. Lo que parece bastante claro es que el acceso abierto no sólo va a producir que la ciencia avance de otra manera, sino que aumente la velocidad a la que se mueve [58].

\section{Bibliografía}

1. Orden $679 / 2009$ de 19 de febrero, por la que se establecen las bases reguladoras de ayudas a programas de actividades de I+D entre grupos de investigación de la Comunidad de Madrid y convocatoria en tecnologías cofinanciada con Fondo Social Europeo. BOCM 53, de 4 de mayo de 2009, p. 13.

2. Resolución del presidente del Consejo Superior de Investigaciones Científicas por la que se convocan ayudas para apoyar las líneas de I+D en el programa de creación y consolidación de grupos de investigación del Consejo Superior de Investigaciones Científicas en la Comunidad de Madrid. BOCM 119, de 20 de mayo de 2008, p. 122-7.

3. Resolución del rector de la Universidad Carlos III de Madrid, de 1 de julio de 2009, por la que se resuelve, en el marco del programa propio de investigación, la convocatoria de ayudas para incrementar la presencia de los institutos y grupos de investigación de la Universidad Carlos III de Madrid en Internet. URL: https://www.uc3m.es/ portal/page/portal/investigacion/programas_convocatorias/ programa_vicerrectorado_09/ayudas_web/Resolucion $\% 20$ Web.pdf. [25.11.2009].

4. Resolución de 20 de mayo de 2008 , del rector, por la que se establece la convocatoria de ayudas para la realización de proyectos de investigación del programa de creación y consolidación de grupos de investigación. BOCM 127, de 20 de mayo de 2008.

5. Bayley CHW. Open access bibliography. Liberating scholarly literature with e-prints and open access journals. Washington, DC: Association of Research Libraries; 2005. URL: http:// www.digital-scholarship.org/oab/oab.pdf. [25.11.2009].

6. Buñuel-Âlvarez JC. Revista Pediatría de Atención Primaria: en camino hacia el acceso abierto. Rev Pediatr Aten Primaria 2009; 11: 203-6.

7. San José-Montalvo B. Acceso abierto (open access), un modelo necesario de comunicación científica. Rev Pediatr Aten Primaria 2009; 11: 299-311.

8. Sánchez-Martín FM, Millán-Rodríguez F, Villavicencio Marvrich $\mathrm{H}$. La iniciativa open access (OAI) en la literatura científica. Actas Urol Esp 2009; 33: 732-40.

9. Sanz-Valero J, D’Agostino MJ, Castiel LD, Veiga de Cabo J. La iniciativa open access, una visión de conjunto. Medicina y Seguridad del Trabajo 2007; 53: 5-10.

10. González de Dios J, Sempere AP, Aleixandre-Benavent R. Las publicaciones biomédicas en España a debate (II). Las revoluciones pendientes y su aplicación a las revistas neurológicas. Rev Neurol 2007; 44: 101-12-

11. Abad-García MF, González-Teruel A, Martínez-Catalán C. Acceso abierto y revistas médicas españolas. Med Clin (Barc) 2006; 127: 456-64.

12. Guerrero R, Piqueras M. Open access. A turning point in scientific publication. Int Microbiol 2004; 7: 157-61.

13. Berlin Declaration on open access to knowledge in the sciences and humanities. Berlin, 2003. URL: http://www.zim. mpg.de/openaccess-berlin/berlindeclaration.htm. [25.11.2009].

14. Bethesda Statement on Open Access Publishing. URL: http://www.earlham.edu/ peters/fos/bethesda.htm. [25.11.2009].

15. Budapest Open Access Initiative. URL: http://www.soros. org/openaccess/read.shtml. [25.11.2009].

16. Association of Research Libraries. Serial Expenditures in ARL Libraries. URL: http://www.arl.org/bm doc/monser06. pdf. [25.11.2009].

17. Sherpa/Juliet. Selected research funders' grant expenditure. URL: http://www.sherpa.ac.uk/juliet/financialstats. [25.11.2009]. 
18. Sherpa/Juliet. Research funders' open access policies. URL: http://www.sherpa.ac.uk/juliet/. [25.11.2009].

19. National Institutes of Health. Policy on enhancing public access to archived publications resulting from NIH-Funded Research. URL: http://grants.nih.gov/grants/guide/noticefiles/NOT-OD-05-022.html. [25.11.2009].

20. SPARC. Federal Research Public Access Act of 2009 (S.1373). URL: http://www.arl.org/sparc/advocacy/frpaa. [25.11.2009].

21. OECD. Principles and guidelines for access to research data from public funding. URL: http://www.oecd.org/ dataoecd/9/61/38500813.pdf. [25.11.2009].

22. IFLA/UNESCO Internet Manifesto Guidelines. Septiembre 2006. URL: http://archive.ifla.org/faife/policy/iflastat/ Internet-ManifestoGuidelines.pdf. [25.11.2009].

23. European Commission. Open access in FP7. URL: ftp://ftp. cordis.europa.eu/pub/fp7/docs/open-access-pilot_en.pdf. [25.11.2009].

24. Silio T. Fundamentos tecnológicos del acceso abierto: open archives initiative y Open Archival Information System. Prof Inf 2005; 14: 365-8.

25. PADICAT. Patrimonio Digital de Catalunya. URL: http:// www.padicat.cat. [25.11.2009].

26. Biblioteca Virtual de Prensa Histórica. URL: http:// prensahistorica.mcu.es. [25.11.2009].

27. EMD. Euskal Memoria Digitala-Memoria Digital Vasca. URL: http://www.memoriadigitalvasca.es. [25.11.2009].

28. OPLex: colección de normativa de carreteras y ferrocarriles. URL: http://infodigital.opandalucia.es/oplex. [25.11.2009].

29. ArXiv. URL: http://arxiv.org. [25.11.2009].

30. E-Prints in Library and Information Science. URL: http:// eprints.rclis.org. [25.11.2009].

31. Elsevier. What right do I retain as an author? URL: http:// www.elsevier.com/wps/find/supportfaq.cws_home/ rightsasanauthor. [25.11.2009].

32. RoMEO Publisher copyright policies \& self-archiving. URL: http://www.sherpa.ac.uk/romeo.php. [25.11.2009].

33. RoMEO Publisher copyright policies \& self-archiving. URL http://www.sherpa.ac.uk/romeo/statistics.php. [25.11.2009].

34. DULCINEA. URL: http://www.accesoabierto.net/dulcinea/. [25.11.2009].

35. Melero R, Abad-García MF, Abadal E, Rodriguez-Gairin JM. DULCINEA: Copyright Policies and Type of Access to Spanish Scientific Journals. ELPUB 2009: 13th International Conference on Electronic Publishing: Rethinking Electronic Publishing: Innovation in Communication Paradigms and Technologies.

36. Keefer A. Los repositorios digitales universitarios y los autores. Anales de Documentación 2007; 10: 205-14.

37. Swan A, Dixon S. Open access self archiving: an author study. Corn-wal, Key perspectives limited, 2005. URL: http://www.keyperspectives.co.uk/openaccessarchive/ reports/Open\%20Ac cess\%20II\%20(author\%20survey\%20 on\%20self\%20archiving)\%202005.pdf. [25.11.2009].

38. Rowlands I, Nicholas D. New journal publishing models: an international survey of senior researchers. Publishers Association and the International Association of STM Publishers, 2005. URL: http://www.ucl.ac.uk/ciber/ ciber_2005_survey_final.pdf. [25.11.2009].
39. OPENDOAR. The Directory of Open Access Repositories. URL: http://www.opendoar.org. [25.11.2009].

40. Aphasiology Archive. URL: http://aphasiology.pitt.edu. [25.11.2009]

41. E-ms. Open archive in social medicine and related fields. URL: http://e-ms.cilea.it. [25.11.2009].

42. CBCRA. Canadian Breast Cancer Research Alliance open access archive. URL: https://researchspace.library.utoronto. $\mathrm{ca} / \mathrm{handle} / 1807.1 / 1$. [25.11.2009]

43. PubMed Central. Free archive of life sciences journals. URL: http://www.ncbi.nlm.nih.gov/pmc. [25.11.2009].

44. UK PubMed Central. Free archive of life sciences journals. URL: http://ukpmc.ac.uk. [25.11.2009].

45. PubMed Central Canada. Free archive of life sciences journals. URL: http://pubmedcentralcanada.ca. [26.11.2009].

46. BuscaRepositorios. URL: http://www.accesoabierto.net/ repositorios. [25.11.2009].

47. Repositorio de la sociedad española de neuropsiquiatría. URL: http://documentacion.aen.es. [25.11.2009].

48. Digital CSIC: acceso abierto a documentos digitales. URL: http://digital.csic.es. [25.11.2009].

49. Alfonso A. Revistas biomédicas españolas: relevancia académica, impacto científico o factor de impacto. Rev Neurol 2009; 48: 113-6.

50. González de Dios J, Valderrama-Zurián JC, GonzálezAlcaide G, Sempere AP, Bolaños-Pizarro M, AleixandreBenavent R. Aproximación al facto de impacto de las revistas biomédicas en ciencias neurológicas: estudio de los indicadores en el Journal Citation Reports-Science Citation Index 2006. Rev Neurol 2009; 48: 117-28.

51. Sánchez-Andrés JV, Viguera C. Análisis: factor de impacto y comunicación científica. Rev Neurol 2009; 49: 57.

52. Lawrence $\mathrm{S}$. Free online availability substantially increases a paper's impact, Nature, 2001. URL: http://www.nature.com/ nature/debates/e-access/Articles/lawrence.html. [25.11.2009]

53. Harnad S, Brody, T. Comparing the impact of open access $(\mathrm{OA})$ vs. non-OA articles in the same journals, D-Lib Magazine, 2004, 10 (6). URL: http://www.dlib.org/dlib/ june04/harnad/06harnad.html. [25.11.2009].

54. Antelman K. Do open-access articles have a greater research impact? College and Research Libraries 2004; 65: 372-82.

55. Eysenbach G. Citation advantage of open access articles. PLoS Biol 2006; 4: e157. doi:10.1371/journal.pbio.0040157.

56. Harnad S. The access/impact problem and the green and gold roads to open access: an update. Serials Review 2008; 34: 36-40.

57. Hajjem CS, Harnad S, Gingras Y. Ten-year cross-disciplinary comparison of the growth of open access and how it increases research citation impact. IEEE Data Engineering Bulletin 2005; 28: 39-47. URL: http://eprints.ecs.soton. ac.uk/12906. [25.11.2009].

58. Swan A. El acceso abierto y el progreso de la ciencia. Análisis Madrid+D. 2008. URL: http://www.madrimasd. org/informacionIdi/analisis/analisis/analisis.asp?id= 35062\&sec $=17$. [25.11.2009].

\section{Self-archiving of biomedical papers in open access repositories}

Introduction and development. Open-access literature is digital, online, free of charge, and free of most copyright and licensing restrictions. Self-archiving or deposit of scholarly outputs in institutional repositories (open-access green route) is increasingly present in the activities of the scientific community. Besides the benefits of open access for visibility and dissemination of science, it is increasingly more often required by funding agencies to deposit papers and any other type of documents in repositories. In the biomedical environment this is even more relevant by the impact scientific literature can have on public health. However, to make self-archiving feasible, authors should be aware of its meaning and the terms 
in which they are allow to archive their works. In that sense, there are some tools like Sherpa/RoMEO or DULCINEA (both directories of copyright licences of scientific journals at different levels) to find out what rights are retained by authors when they publish a paper and if they allow to implement self-archiving. PubMed Central and its British and Canadian counterparts are the main thematic repositories for biomedical fields. In our country there is none of similar nature, but most of the universities and CSIC, have already created their own institutional repositories.

Conclusion. The increase in visibility of research results and their impact on a greater and earlier citation is one of the most frequently advance of open access, but removal of economic barriers to access to information is also a benefit to break borders between groups.

Key words. Biomedical journals. Open access. Repositories. Self-archiving. 